\title{
Doppler imaging of stellar surface structure
}

\section{$X V$. A possible detection of differential rotation and local meridional flows on the rapidly-rotating giant HD $218153=\mathrm{KU}$ Pegasi}

\author{
M. Weber ${ }^{\star}$ and K. G. Strassmeier* \\ Astrophysikalisches Institut Potsdam, An der Sternwarte 16, 14482 Potsdam, Germany \\ e-mail: kstrassmeier@aip.de \\ Received 21 February 2001 / Accepted 11 April 2001

\begin{abstract}
Time-resolved Doppler images of the rapidly rotating, but long-period ( 25 days), giant KU Pegasi show several cool low-to-medium latitude spots as well as an asymmetric polar feature. The average spot temperature is about $700 \mathrm{~K}$ below the photospheric temperature of $4700 \mathrm{~K}$. KUPeg is one of the most massive, and currently the most evolved, late-type star with a Doppler image. We obtained two independent images from two consecutive stellar rotations covering 50 nights with a total of 43 spectra. From a cross-correlation analysis of the two maps, we detect systematic longitudinal and latitudinal shifts that we tentatively interpret as latitude-dependent differential rotation and local meridional flows, respectively. The differential-rotation pattern is more complex than on the Sun, but on average in the sense that the poles rotate slower than the stellar equator, i.e. in the same direction and also of the same order than on the Sun. The latitudinal shifts are of the order of $0.4^{\circ}$ day ${ }^{-1}$ towards the stellar pole and occur at longitudes of around $40^{\circ}$ and $330^{\circ}$. The residual $\mathrm{H} \alpha$ profiles show a stationary emission component at rest wavelength and a blue-shifted absorption. The latter suggests an outward pointed velocity field with a flow velocity of approximately $35 \mathrm{~km} \mathrm{~s}^{-1}$.
\end{abstract}

Key words. stars: activity - stars: atmospheres - stars: imaging - stars: individual: HD 218153 - stars: late-type

\section{Introduction}

HD $218153=$ KUPeg $\left(\alpha=23^{\mathrm{h}} 05^{\mathrm{m}} 29^{\mathrm{s}}, \delta=26^{\circ} 00^{\prime} 33^{\prime \prime}\right.$, 2000.0, $\left.V=7^{\mathrm{m}} \cdot 61-7^{\mathrm{m}} \cdot 72\right)$ is a G9-K0 giant with strong Ca II H \& K emission (Bidelman 1983), a projected rotational velocity of $v \sin i=29 \mathrm{~km} \mathrm{~s}^{-1}$ (Fekel 1997), and a photometric period of approximately 24 days (Strassmeier \& Hall 1988) which was interpreted to be the stellar rotation period.

Despite its long rotational period the star ranks near the top in ultraviolet emission-line strength compared to other late-type giants (De Medeiros et al. 1992) (only FK Comae is significantly more active). Its optical spectrum shows only a weak lithium feature in the $6707-\AA$ range - Barrado y Navascues et al. (1998) report $16 \mathrm{~m} \AA$ and Strassmeier et al. (2000) $13 \mathrm{~m} \AA$, which suggests that the star is in the expanding post-main-sequence evolutionary stage and not a contracting pre-main-sequence star.

\footnotetext{
Send offprint requests to: M. Weber, e-mail: mweber@aip.de

* Visiting Astronomer, National Solar Observatory, National Optical Astronomy Observatories, and Kitt Peak National Observatory, operated by the Association of Universities for Research in Astronomy, Inc. under contract with the National Science Foundation.
}

De Medeiros et al. (1992) detected small radial-velocity variations and reported a preliminary spectroscopic orbit with a period of 1411 days ( $\approx 4$ years). Such a long orbital period implies that the star had evolved effectively like a single star similar to HD 51066 (=BM Cam; Strassmeier et al. 1998, Paper VIII in this series) and thus may be compared with a group of truly single, rapidly-rotating giants. Such a group of evolved active stars was first identified by F. C. Fekel and collaborators (see e.g. Fekel \& Balachandran 1993) from spectra with unusually strong lithium lines at $6708 \AA$, despite their evolutionary status. At an age of several hundred Megayears one would expect that the mixing in the outer convective envelope was thorough enough so that most lithium was burned when it was getting to close to the bottom of the convective envelope. How these rapidly-rotating giants maintained a primordial level of lithium on their surface still remains unanswered. Since KU Peg does not show significant lithium on its surface, actually $[\mathrm{Li} / \mathrm{H}]<[\mathrm{Li} / \mathrm{H}]_{\odot}$, but otherwise is a rapidly-rotating extremely active giant, it may represent a border case for stellar convection and light-element dredge-up models.

A brief discussion of the star's photometric history and our APT (automatic photoelectric telescope) data 
prior to 1996 was given by Strassmeier et al. (1999a). The relatively small light and color amplitudes of $\Delta V \approx$ $0{ }^{\mathrm{m}} 03-0^{\mathrm{m}} 07$ and $\Delta(V-I) \leq 0{ }^{\mathrm{m}} 03$ in $1996-98$ suggested that either only a small fraction of the surface was covered with spots or that the spot distribution was relatively symmetric. Our line profile data show the typical bumpsignatures from cool spots and thus verify the existence of an asymmetric spot component at that time.

In this paper, we present a series of 43 moderately high-resolution spectra in the 6430 - $\AA$ region taken over a two month period in late 1996 through early $1997 . V(R I)_{\mathrm{C}}$ photometry was gathered continuously from fall 1996 until now. The combination of these data is used to study the spatial surface distribution of the spots on KU Peg and its short-term variations. The spectroscopic observations for Doppler imaging span a little more than two stellar rotations and are used to derive two independent surface maps. The instrumentation and the data reductions are described in Sect. 2 while the stellar properties relevant for the Doppler-imaging analysis are determined in Sect. 3. $\mathrm{H} \alpha$ spectra from 1998 are also presented in this section. The Doppler maps from three spectral regions and for the two stellar rotations are derived in Sect. 4. Finally, we derive a latitude-dependent differential rotation law from a cross correlation of the two consecutive maps and discuss its relation with the Sun and other active stars.

\section{Observations}

Spectroscopic observations were obtained at the National Solar Observatory (NSO) with the McMath-Pierce main telescope using the stellar spectrograph from October 31, 1996 to January 8,1997 . The $800 \times 800$-pixel TI CCD (TI-4 chip, $15 \mu$ pixels) allowed for a resolving power of $\approx 40000$ and a useful wavelength range of about $45 \AA$ around $6430 \AA$. Two additional spectra in the $6430-\AA$ region plus one spectrum in the $\mathrm{H} \gamma$ region, along with corresponding reference spectra, were obtained at Kitt Peak National Observatory (KPNO) with the coudé feed telescope in December 1997/January 1998. A very similar $800 \times 800$ TI CCD (TI-5 chip, $15 \mu$ pixels) was used in combination with grating A, camera 5, and the long collimator. It allowed for a resolving power of 38000 and 22000 in the red and the blue-wavelength regions, respectively, with a useful wavelength range of between 50-80 . Two more spectra in the $6500-\AA$ region included $\mathrm{H} \alpha$ and were obtained with the same instrumental set-up at the coudé feed telescope in April 1998 but utilized the $3096 \times 1024$ CCD (F3KB chip, $15 \mu$ pixels). The resolving power was 28000 , and the useful wavelength range was $300 \AA$. Table 1 is a summary of the spectroscopic observations. Spectra taken after the second rotational cycle in early 1997 and spectra with signal-tonoise ratios below 100:1 are not used for Doppler imaging (these spectra are marked with a dash in Table 1).
Table 1. Spectroscopic log and radial velocity data $\left(v_{\mathrm{rad}}\right)$.

\begin{tabular}{|c|c|c|c|c|c|}
\hline $\begin{array}{l}\text { HJD } \\
(24+)\end{array}$ & $\begin{array}{r}\text { Phase } \\
\left({ }^{\circ}\right)\end{array}$ & $\begin{array}{l}v_{\mathrm{rad}} \\
\quad\left(\mathrm{km} \mathrm{s}^{-}\right.\end{array}$ & $\begin{array}{l}\sigma_{\text {rad }} \\
-1)\end{array}$ & $\begin{array}{c}\text { Rotation } \\
\text { cycle }\end{array}$ & $\begin{array}{l}\text { Tele- } \\
\text { scope }\end{array}$ \\
\hline 50391.770 & 90.4 & -77.5 & 1.5 & map 1 & NSO \\
\hline 50392.661 & 103.3 & -82.0 & 2.7 & - & NSO \\
\hline 50393.748 & 119.0 & -75.8 & 1.9 & map 1 & NSO \\
\hline 50394.781 & 133.9 & -78.5 & 1.8 & map 1 & NSO \\
\hline 50395.602 & 145.7 & -80.5 & 2.0 & map 1 & NSO \\
\hline 50396.648 & 160.8 & -78.5 & 2.0 & map 1 & NSO \\
\hline 50398.713 & 190.4 & -82.4 & 2.3 & - & NSO \\
\hline 50400.629 & 218.2 & -81.1 & 1.6 & map 1 & NSO \\
\hline 50401.630 & 232.6 & -79.6 & 2.4 & - & NSO \\
\hline 50404.641 & 276.1 & -83.2 & 2.0 & map 1 & NSO \\
\hline 50405.617 & 290.1 & -81.8 & 2.5 & map 1 & NSO \\
\hline 50406.623 & 304.7 & -80.9 & 1.9 & map 1 & NSO \\
\hline 50408.624 & 333.5 & -77.3 & 3.0 & map 1 & NSO \\
\hline 50409.617 & 347.8 & -82.0 & 2.3 & map 1 & NSO \\
\hline 50411.618 & 16.7 & -79.9 & 1.7 & map 1 & NSO \\
\hline 50412.611 & 31.0 & -80.9 & 1.8 & map 1 & NSO \\
\hline 50413.609 & 45.4 & -81.2 & 1.7 & map 1 & NSO \\
\hline 50415.610 & 74.3 & -80.1 & 1.7 & map 1 & NSO \\
\hline 50416.623 & 88.9 & -80.5 & 1.5 & map 1 & NSO \\
\hline 50417.710 & 104.4 & -78.9 & 2.3 & - & NSO \\
\hline 50418.683 & 118.6 & -80.4 & 1.2 & map 2 & NSO \\
\hline 50419.597 & 131.8 & -80.2 & 1.6 & map 2 & NSO \\
\hline 50420.607 & 146.4 & -80.3 & 1.8 & map 2 & NSO \\
\hline 50421.690 & 162.0 & -79.4 & 2.1 & map 2 & NSO \\
\hline 50422.602 & 175.1 & -79.6 & 1.3 & map 2 & NSO \\
\hline 50423.609 & 189.7 & -80.6 & 1.5 & - & NSO \\
\hline 50424.701 & 205.4 & -82.8 & 1.3 & map 2 & NSO \\
\hline 50425.674 & 219.4 & -79.3 & 1.7 & map 2 & NSO \\
\hline 50426.693 & 234.1 & -80.2 & 2.2 & map 2 & NSO \\
\hline 50429.661 & 276.9 & -79.4 & 2.1 & map 2 & NSO \\
\hline 50430.682 & 291.7 & -79.6 & 1.5 & map 2 & NSO \\
\hline 50431.569 & 304.5 & -81.7 & 2.0 & map 2 & NSO \\
\hline 50432.603 & 319.4 & -80.8 & 1.6 & map 2 & NSO \\
\hline 50433.593 & 333.7 & -81.5 & 1.7 & - & NSO \\
\hline 50434.599 & 348.2 & -81.4 & 2.1 & map 2 & NSO \\
\hline 50436.645 & 17.6 & -80.9 & 1.7 & - & NSO \\
\hline 50438.592 & 45.7 & -80.4 & 2.0 & map 2 & NSO \\
\hline 50440.618 & 75.0 & -79.8 & 2.3 & map 2 & NSO \\
\hline 50441.604 & 89.3 & -80.0 & 2.4 & - & NSO \\
\hline 50445.594 & 146.9 & -80.0 & 2.2 & - & NSO \\
\hline 50446.595 & 161.3 & -81.3 & 2.5 & - & NSO \\
\hline 50450.682 & 220.0 & -81.3 & 1.9 & - & NSO \\
\hline 50457.625 & 320.4 & -81.5 & 1.8 & - & NSO \\
\hline 50821.638 & 170.3 & -78.7 & 1.1 & - & KPNO \\
\hline 50826.670 & 243.0 & -80.0 & 1.0 & - & KPNO \\
\hline 50828.628 & 271.4 & -78.5 & 1.6 & - & KPNO \\
\hline 50918.011 & 120.6 & -79.0 & 1.0 & - & KPNO \\
\hline 50921.003 & 163.4 & -80.3 & 0.7 & - & KPNO \\
\hline
\end{tabular}

All data were reduced using $\operatorname{IRAF}^{1}$ and included bias subtraction, flat fielding, cosmic-ray removal, and an optimal aperture extraction. The exposure level obtained with an integration time of $30 \mathrm{~min}$ corresponds to a

\footnotetext{
${ }^{1}$ Image Reduction and Analysis Facility distributed by NOAO/KPNO.)
} 

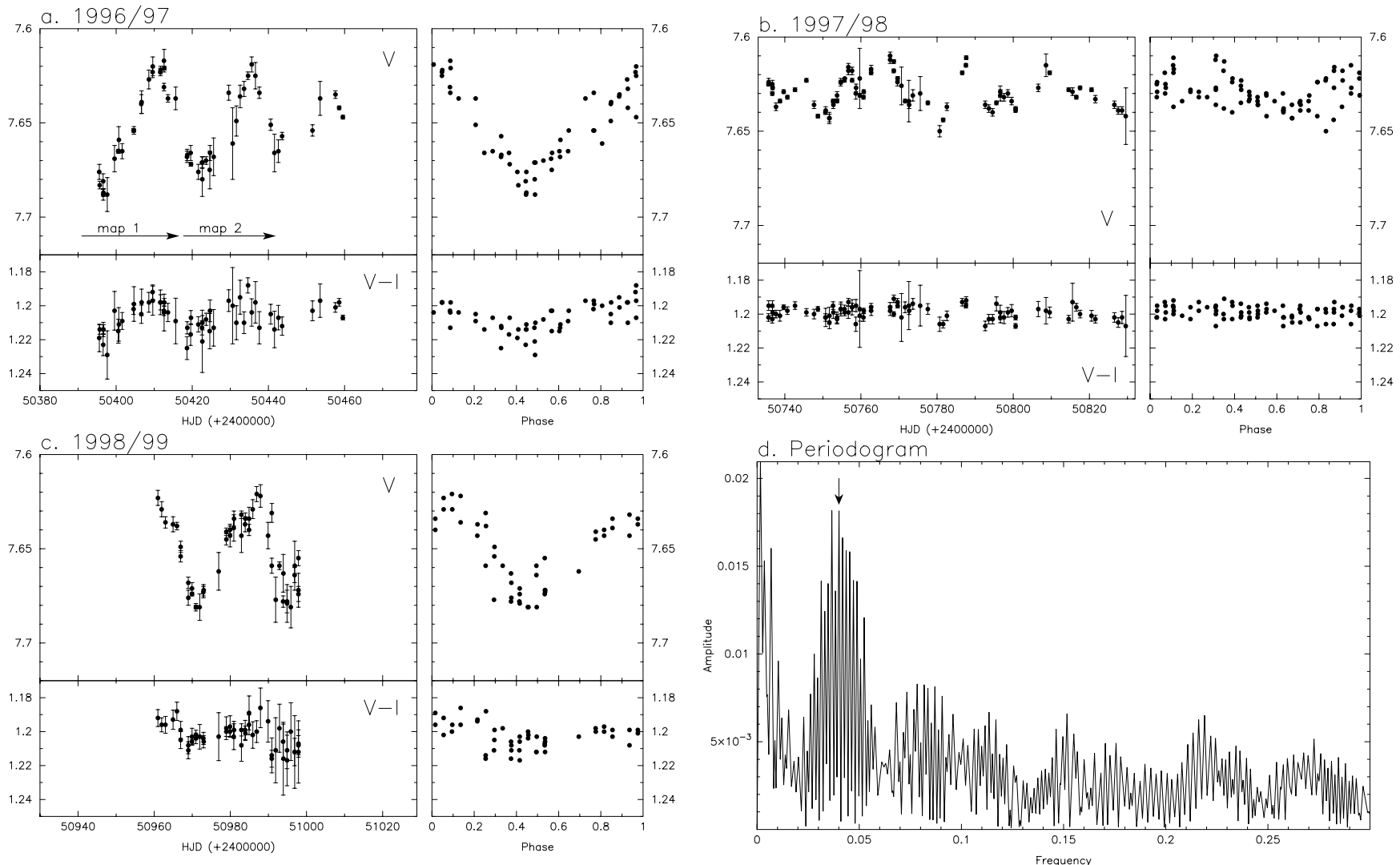

Fig. 1. Three years of photometry of KU Peg. a) The 1996/97 APT data versus Julian date and versus phase calculated with the ephemeris in Eq. (1), b) and c) the same for the 1997/98 and 1998/99 seasons, and d) the periodogram for the combined 1996-99 APT data.

signal-to-noise ratio of approximately 150:1. Usually twenty flat-field exposures with a Tungsten reference lamp were taken at the beginning of the night and again at the end of the night. These forty flat fields were co-added and used to remove the pixel-to-pixel variations in the stellar spectra. Spectra of bright radial-velocity standards were obtained several times throughout the night to ensure an accurate wavelength calibration. Radial velocities for the 1996/97 NSO data and the two KPNO spectra from JD 2450826 and JD 2450828 were derived from cross correlations with spectra of the IAU velocity standard $\alpha$ Ari (K2III, $v_{\mathrm{r}}=-14.3 \mathrm{~km} \mathrm{~s}^{-1}$ ). The one spectrum from JD 2450821 was cross correlated with $\beta$ Gem (K0III, $v_{\mathrm{r}}=3.3 \mathrm{~km} \mathrm{~s}^{-1}$ ). $16 \operatorname{Vir}\left(\mathrm{K} 0.5 \mathrm{III}, v_{\mathrm{r}}=35.7 \mathrm{~km} \mathrm{~s}^{-1}\right.$ ) was used for the remaining spectra.

The photometric data in this paper were obtained with the Amadeus 0.75-m automatic photoelectric telescope (APT), part of the University of Vienna twin APT at Washington Camp in southern Arizona (Strassmeier et al. 1997). The observations were made differentially with respect to HD 218610 as the comparison star $\left(V=7^{\mathrm{m}} \cdot 64\right.$, $\left.V-R_{\mathrm{C}}=0 .{ }^{\mathrm{m}} 69, V-I_{\mathrm{C}}=1.30\right)$ and SAO 91066 as the check star. The data cover the time interval JD $2450395-$ 459 (54 data points, season 1996/97), JD 2450740-828 (80 data points, season 1997/98), and JD 2450960-998
(47 data points, season 1998/99). All photometry was transformed to match the Johnson-Cousins $V(R I)_{\mathrm{C}}$ system.

\section{Stellar properties of KU Peg}

\subsection{The rotation period}

We applied the multiple period search program PERIOD98 (Sperl 1998) in single-period mode and use its Fourier option to determine the photometric period of KUPeg (Fig. 1d). The application of this program to photometry of spotted stars was recently discussed in Strassmeier et al. (1999a) and we refer the reader to this paper. The fit with the smallest $\chi^{2}$ was obtained with a period of 24 d. $6 \pm 0.5$ for the $1996 / 97$ data, $25^{\mathrm{d}} 0 \pm 1.1$ in $1997 / 98$, and $23.9 \pm 0.8$ for the $1998 / 99$ data (Figs. 1a-c).

By early 1997/98 the amplitude of the $V$-lightcurve had dropped from 0.07 in late 1996 to $\approx 0.03$ in late 1997, and had risen again to 0.06 in 1998. The Fourier analysis of the $1997 / 98 \mathrm{~V}$ data still shows a peak near the same frequency as in $1996 / 97$ but an alias at $f=0.1$ $c / d$ appears with an even stronger amplitude than the true photometric period. Such a reduced rotational-modulation signal suggests that the active regions that were visible in 


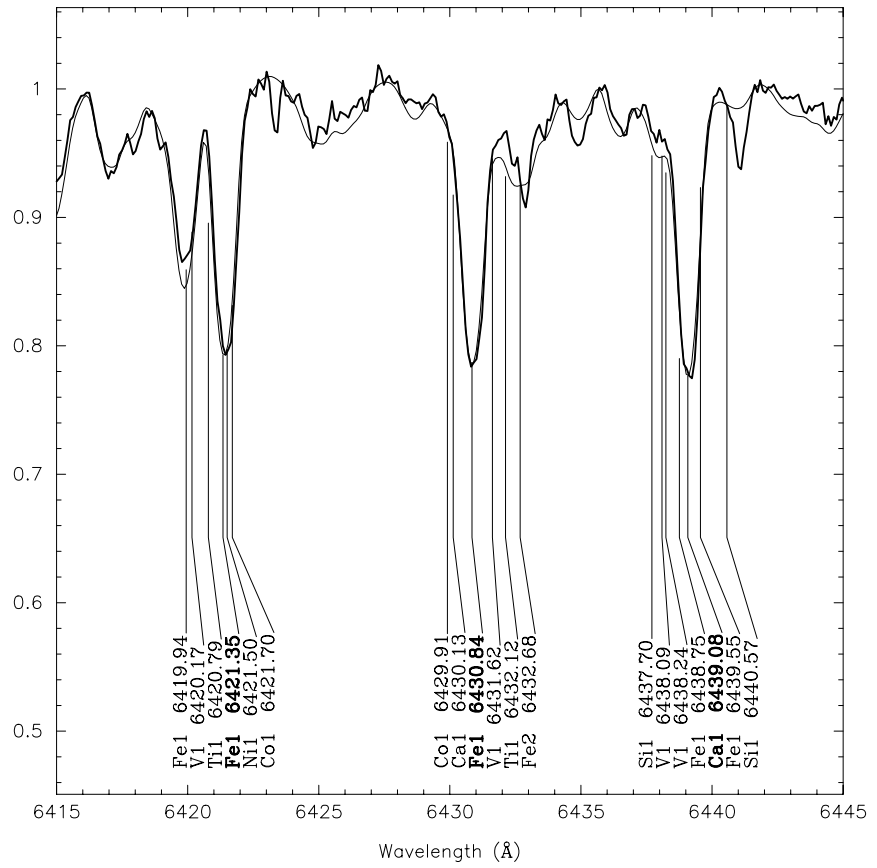

Fig. 2. A comparison of a spectrum of KUPeg (thick line) with a shifted and spun-up spectrum of the G9II-III standard star HD 76294 (thin line). Identified are the spectral lines that are used in the Doppler-imaging analysis in Sect. 4 (a boldface wavelength distinguishes the main mapping line from the blends included).

1996/97 had significantly decreased in size and/or contrast by late 1997. The lightcurves of the two seasons (Figs. 1a and b) consistently show a minimum at around phase 0.5 but the phased 1997/98-season data appear as a doublehumped light curve.

The final period is obtained from the combined data set and is of higher precision due to the longer time range. We obtain $24.96 \pm 0.04$, which we interpret to be the stellar rotation period and use it to calculate the phases for all spectroscopic and photometric data in this paper:

$\mathrm{HJD}=2450385.5+24.96 \pm 0.04 \times E$.

The strongest peak in Fig. 1d $\left(0.002\right.$ day $\left.^{-1}\right)$ is an alias resulting from the time gap between the three observing seasons. The other peak of comparable size (27.33 days) is basically due to the same reason.

\subsection{Effective temperature and spectral classification}

The effective temperature of KUPeg is estimated in several ways, first from a comparison of the observed $V-I_{\mathrm{C}}$ color with synthetic colors from the ATLAS-9 model atmospheres (Kurucz 1993). The bluest $(V-I)_{\mathrm{C}}$ value of $1.17 \pm 0{ }^{\mathrm{m}} 01$ is observed at times of lightcurve maximum (Fig. 1) and is adopted to best represent the "unspotted" intrinsic color of the photosphere. This value is different to the long-term average due to the color variations from spots of up 0.03 . Comparing this $V-I$ value with the grid of synthetic ATLAS-9 colors in the range $\log g=2.0$ to
3.0 and metal abundances of solar to 1.0 dex below solar, we find a temperature of $4700 \pm 150 \mathrm{~K}$. This compares very well with the $4700 \mathrm{~K}$ from the observed $B-V$ of $1 . \mathrm{m} 13$ and the calibration for a bright giant from Bell \& Gustafsson (1989). Similar temperatures are obtained from the colortemperature calibration of Flower (1996) (4622 K), as well as from the combined G9II, G9II-III and G9III temperatures listed in Dyck et al. (1998) $(4686 \pm 192 \mathrm{~K})$, for a G9III star in van Belle et al. (1999) (4679 K), and for a K0 giant in Bell \& Gustafsson (1989) (4820 K).

Another method for estimating the effective temperature is to use the temperature dependence of spectral lines. Using the calibration for giant stars from spectral line-depth ratios from Strassmeier \& Schordan (2000), we calculated the $V-I$ colors and the effective temperatures for 11 line-ratios. The resulting average effective temperature was $4835 \pm 62 \mathrm{~K}$, in good agreement with the values derived with other methods. The average $V-I$-color of $1.06 \pm 0.05$ is 0.11 smaller than the observed value, which shows that the large interstellar absorption correction we apply in the next section is indeed of the right order of magnitude.

In order to compare the spectrum of KU Peg numerically with spectra of Morgan-Keenan standard stars, we make use of a computer program originally designed by Barden (1985). The standard-star spectra are Fourier transformed and subtracted from a representative KU Peg spectrum. The resulting difference spectra are iteratively minimized by changing the relative continuum intensity, the rotational broadening, and the radial velocity of the KU-Peg spectrum by means of a weighted $\chi^{2}$ minimization. With this procedure, we found the best fit with a spectral type of G9 to K0 III and a (preliminary) rotational velocity of $v \sin i=28 \pm 2 \mathrm{~km} \mathrm{~s}^{-1}$. We note that both standards, $\beta$ Gem (K0III) and HD 76294 (G9II-III), fitted our KUPeg spectra equally well (the latter fit is shown in Fig. 2).

To obtain an independent estimate for the luminosity class of KUPeg, we use the luminosity-sensitive Sr II 4077- $\AA$ line and compare KUPeg with $\alpha$ Ari (K2III), $\beta$ Gem (K0III), Ł Cnc (G8II), and 55 Cam (G8II). Again, the program of Barden (1985) was used to minimize the residual spectra. Because the Sr II 4077 line of KU Peg is weaker than in the two available G8II spectra, but only slightly stronger than in the $\beta$ Gem spectrum, we estimate KUPeg to have a luminosity class of II-III and therefore a most likely spectral classification of G9-K0 II-III.

\subsection{Radius, luminosity, and mass}

The rotational period and the projected rotational velocity determine the minimum stellar radius to be $R \sin i=$ $13.8 \pm 0.5 R_{\odot}$. Using an inclination angle of $50 \pm 10^{\circ}-$ that will be derived later in Sect. 4.2 - the most likely stellar radius of $\mathrm{KU} \mathrm{Peg}$ is $18_{-3}^{+4} R_{\odot}$. This is not in agreement with the $13.9 \pm 0.3 R_{\odot}$ for an average K0III star according to van Belle et al. (1999), and just barely in agreement 
with the $15 R_{\odot}$ according to the Landolt-Börnstein tables (Schmidt-Kaler 1982) but more likely indicates that the star is larger than a regular K0 giant. Using above radius and an effective temperature of $4700 \pm 150 \mathrm{~K}$, the bolometric magnitude of KU Peg is $M_{\mathrm{bol}}=-0.74_{-0.57}^{+0.55}$.

The Hipparcos parallax of $5.33 \pm 0.91$ milli-" (ESA 1997) puts KU Peg in a distance of $188_{-28}^{+38} \mathrm{pc}$ and, with the brightest $V$ magnitude observed so far, i.e. $V=7^{\mathrm{m}} \cdot 61$ in 1997 (see Fig. 1c), results in an absolute visual brightness of $0.82_{+0.35}^{-0.40}$. An interstellar extinction correction of $A_{V}=0.42$ had been applied, which results from $A_{V}=3.2 E(B-V)$, where $E(B-V)$ is 0.13 using $B-V=1{ }^{\mathrm{m}} 13 \pm 0.02$ (Guetter 1980; Fernie 1983) and $(B-V)_{0}=1 .{ }^{\mathrm{m}} 0$ (Schmidt-Kaler 1982). This agrees with the $A_{V}=0.52$ listed in Guarinos (1992). With a bolometric correction of -0.48 (Flower 1996) the bolometric magnitude is $M_{\mathrm{bol}}=+0^{\mathrm{m}} 34_{+0.34}^{-0.39}$, which is different by one magnitude to the above value from the combination of the minimum stellar radius, the inclination from the Doppler-imaging analysis, and the effective temperature from the spectral classification. Adopting an average bolometric magnitude and a solar value of $+4{ }^{\mathrm{m}} 64$, the luminosity of KU Peg is $97 \pm 45 L_{\odot}$.

A comparison of the position of KUPeg in the $\mathrm{H}-\mathrm{R}$ diagram with the evolutionary tracks of Schaller et al. (1992) interpolated for 0.6 dex below solar metallicity (see Sect. 4.3), suggests a mass of $2.3 \pm 0.3 M_{\odot}$ and an age of approximately 870 Myrs.

\subsection{Orbital period}

De Medeiros et al. (1992) reported the detection of radial velocity variations with a full amplitude of $6 \mathrm{~km} \mathrm{~s}^{-1}$ and calculated a preliminary orbit with the following elements: $P=1411 \pm 26$ days, $T=2447951 \pm 27, e=0.39 \pm 0.14$, $\gamma=-80.4 \pm 0.2 \mathrm{~km} \mathrm{~s}^{-1}, K_{1}=2.5 \pm 0.5 \mathrm{~km} \mathrm{~s}^{-1}$, and $\omega=198 \pm 9^{\circ}$.

The average from our radial velocity measurements from HJD $2450392-457$ is $-80.3 \mathrm{~km} \mathrm{~s}^{-1}$, and agrees very well with the $\gamma$-velocity determined by De Medeiros et al. (1992). Its root mean square is $1.8 \mathrm{~km} \mathrm{~s}^{-1}$ and the average error of our individual velocities is also $1.8 \mathrm{~km} \mathrm{~s}^{-1}$. This values fit the published orbit when a period of 1389 days is used (which is inside the error bounds given by De Medeiros et al. 1992). The phase coverage of our spectra, however, does not allow an independent period determination but verifies the preliminary orbit.

\section{5. $\mathrm{H \alpha}$ line profiles}

Our KPNO spectra from April 1998 included the wavelength region around the $\mathrm{H} \alpha$ line, which is widely used as an activity and velocity indicator for stellar chromospheres (see e.g. Bopp et al. 1988). Our spectra show a broad and asymmetric absorption line with a weak blueshifted emission component (Fig. 3). To extract the $\mathrm{H} \alpha$ contribution from the active part of the chromosphere,
Table 2. Stellar parameters for KU Peg.

\begin{tabular}{ll}
\hline Parameter & Value \\
\hline Spectral type & G9-K0 II-III \\
$\log g$ & $2.5 \pm 0.5$ \\
$T_{\text {eff }}$ & $4700 \pm 150 \mathrm{~K}$ \\
$V_{\max }$ & $7 \cdot 61$ \\
$B-V$ & $1 \cdot 13 \pm 0 \cdot 02$ \\
$\left(V-I_{\mathrm{C}}\right)_{\max }$ & $1^{\mathrm{m}} 17 \pm 0.01$ \\
$v \sin i$ & $28.2 \pm 0.7 \mathrm{~km} \mathrm{~s}^{-1}$ \\
Rotation period $P_{\mathrm{phtm}}$ & $24.96 \pm 0.04$ days \\
Inclination $i$ & $50^{\circ} \pm 10^{\circ}$ \\
Radius $R$ & $18_{-3}^{+4} R_{\odot}$ \\
Equatorial velocity $v_{\text {equ }}$ & $36.8 \mathrm{~km} \mathrm{~s}^{-1}$ \\
Luminosity $L$ & $97 \pm 45 L_{\odot}$ \\
Mass $M$ & $2.3 \pm 0.3 M_{\odot}$ \\
Age & $\approx 870 \mathrm{Myrs}^{-1}$ \\
Microturbulence $\xi$ & $2.0 \mathrm{~km} \mathrm{~s}{ }^{-1}$ \\
Macroturbulence $\zeta_{\mathrm{R}}=\zeta_{\mathrm{T}}$ & $4.0 \mathrm{~km} \mathrm{~s}$ \\
$\log [$ Ca] abundance & $1.0 \mathrm{dex}$ below solar \\
log[Fe] abundance & $0.6 \mathrm{dex} \mathrm{below} \mathrm{solar}$ \\
Distance & $188_{-28}^{+38}$ pc \\
\hline
\end{tabular}

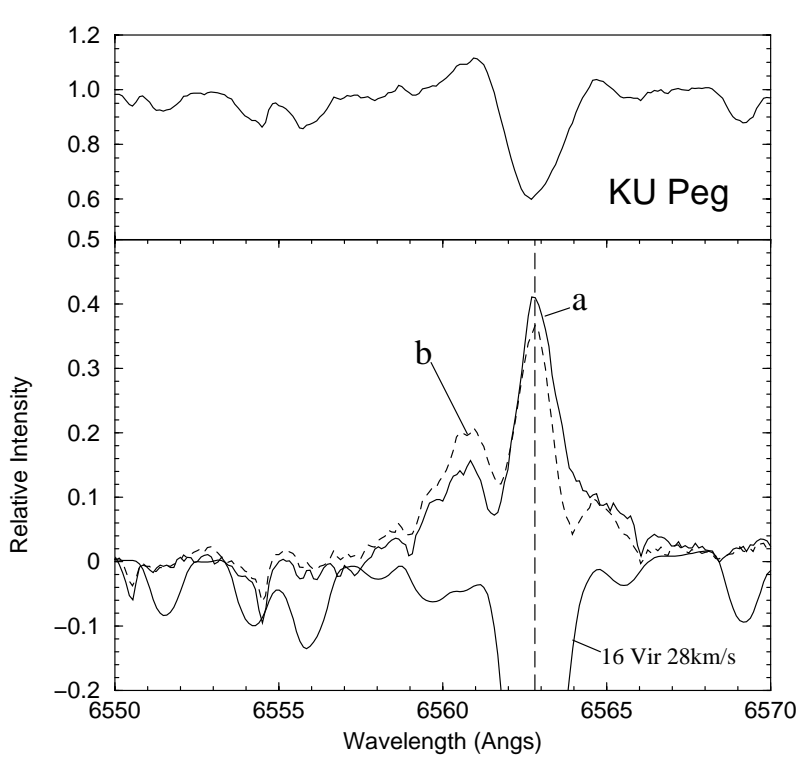

Fig. 3. Residual H $\alpha$ profiles of KU Peg (lower panel) after subtraction of a broadened $\left(v \sin i=28 \mathrm{~km} \mathrm{~s}^{-1}\right)$ spectrum of the inactive reference star 16 Vir. The upper panel plots an unaltered $\mathrm{H} \alpha$ spectrum of KU Peg. The two spectra labeled $a$ and $b$ were taken three days apart.

we subtract the broadened spectrum of the inactive reference star $16 \mathrm{Vir}$ (K0.5 III). To first order, this removes the photospheric and chromospheric flux that is unrelated to the magnetic activity. This subtraction reveals a double-peaked emission profile with unequally strong emission peaks which is typical for very active RS CVn binaries. We assume that the doubled peak results from one emission peak combined with an off-centered absorption line. Using the Gaussian deblending routine in the IRAF splot package, we find that the strong emission peak is centered at the $\mathrm{H} \alpha$ rest wavelength, while the absorption feature appears blueshifted by $-40 \pm 5 \mathrm{~km} \mathrm{~s}^{-1}$ in 
spectrum $a$ (JD 2450918 ) in Fig. 3 and $-30 \pm 5 \mathrm{~km} \mathrm{~s}^{-1}$ in spectrum $b$ (JD 2450921). The full width at half maximum $(F W H M)$ of the Gaussian for the absorption component of these fits is $1.08 \pm 0.05 \AA$, which transforms to $v \sin i=28.6 \pm 1.5 \mathrm{~km} \mathrm{~s}^{-1}$ using the relation described in Strassmeier et al. (1990), and thus seems to originate from near the stellar surface. Although we can not draw more firm conclusions from these two $\mathrm{H} \alpha$ spectra, we do note that the line-profile structure and its asymmetry suggest a complex flow structure in the lower chromosphere and possibly also in the outermost coronal layers, say, one stellar radius above, where hydrogen can be trapped in coronal loops.

\section{Time-series Doppler imaging}

\subsection{The line-profile inversion code}

As for all previous papers in this series, the maps were calculated with the Doppler-imaging code TEMPMAP (Rice et al. 1989; Piskunov \& Rice 1993; Rice 1996). See e.g. Paper X (Strassmeier et al. 1999b) for an updated description of the program and Paper VI (Strassmeier \& Rice 1998) and Rice \& Strassmeier (2000) for numerical tests with artificial data.

Because of the small wavelength coverage of our NSO spectra, we could only use three main mapping lines: Fe I 6421, Fe I 6430 and Ca I 6439 with $\log g f$ values of $-2.23,-2.0$ and +0.47 and lower excitation potentials of $2.279,2.176$ and $2.526 \mathrm{eV}$, respectively. Since all three lines are blended to a certain degree - the $6421-\AA$ region seems to be especially vulnerable - the number of lines which had to be synthesized was 6,6 , and 7 for the 6421,6430 and 6439-line regions, respectively. All these blends are included in the inversion and treated simultaneously with the primary mapping lines but only one spectral region can be handled per solution. We employed a maximumentropy regularization for the Doppler maps presented in this paper, but the program also allows a Tikhonov regularizing functional (for a comparison see, e.g., Piskunov \& Rice 1993). An appropriate set of model atmospheres was taken from the ATLAS-9 CD (Kurucz 1993). All radiation transfer calculations are done under the assumption of LTE.

\subsection{Finetuning the rotational velocity and the inclination of the stellar rotation axis}

Because the Doppler-imaging analysis is sensitive to the rotational velocity and to the inclination of the stellar rotation axis, it can be used to refine these two parameters with higher accuracy than with the method described in Sect. 3 (see also, e.g., Unruh 1996). Changing these two parameters one at a time, while all others are kept constant, yields various values for the misfit between the data and the model $\left(\chi^{2}\right)$. The value of the parameter corresponding to the smallest $\chi^{2}$ is the one we believe is closest to the true value. The variation of $\chi^{2}$ with the inclination
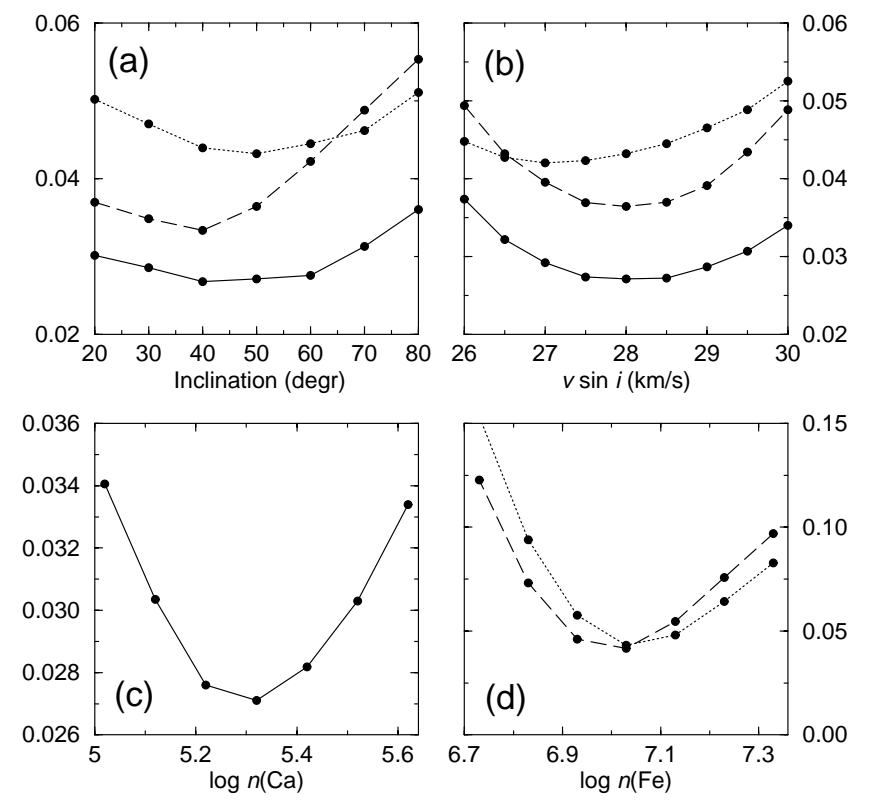

Fig. 4. Optimization of some stellar parameters for Rotation 1. a) The inclination in degrees, $\mathbf{b}$ ) the projected rotational velocity, c) the calcium abundance in units of $\log n(\mathrm{H})=12.0$ and d) the iron abundance. The full line is for the Ca I 6439 line, the dashed line for Fe I 6430, and the dotted line for Fe I 6421. The vertical axis plots $\chi^{2}$ in relative units.

$i$ and the rotational velocity $v \sin i$ are plotted in Figs. $4 \mathrm{a}$ and b, respectively. A minimum is seen in both cases: for the inclination between $40^{\circ}$ and $60^{\circ}$, and for the projected rotational velocity between 27 and $29 \mathrm{~km} \mathrm{~s}^{-1}$. We obtain $v \sin i=28.2 \pm 0.7 \mathrm{~km} \mathrm{~s}^{-1}$ and $i=50^{\circ} \pm 10^{\circ}$ as our final values, according to the unweighted average minimum in Figs. $4 \mathrm{a}$ and $\mathrm{b}$.

\subsection{Finetuning the elemental surface abundances}

To determine approximate elemental abundances, we evaluate the run of the $\chi^{2}$ from a series of inversion solutions with different initial abundances. A straightforward computation of the local line profiles with solar abundances and from model atmospheres with gravities between $\log g=2.0-3.0$, and micro- and macroturbulences between $0.5-4.0 \mathrm{~km} \mathrm{~s}^{-1}$, already indicated a relative underabundance of calcium and iron with respect to the Sun. Our test inversions were thus started with solar abundances and decreased in steps of 0.1 dex (Figs. 4c and d). The transition probabilities, damping constants, and laboratory wavelengths were kept constant at the values specified in Sect. 4.1 and taken partially from the VALD database (Kupka et al. 1999) and our previous papers in this series. We then adopted the abundances that resulted in the least $\chi^{2}$ as the most probable values for the surface abundances, i.e. $\log n(\mathrm{Fe})=7.03$ and $\log n(\mathrm{Ca})=5.32$ (on the $\log n(\mathrm{H})=12.00$ scale). Although an internally consistent set of parameters of high precision, these abundances are of low accuracy because test inversions with different sets of fixed parameters 
Table 3. Table of surface features A-F from the average maps ( $\ell$ longitude, $b$ latitude, $T_{\text {spot }}$ spot temperature).

\begin{tabular}{crrrrrr}
\hline Spot & \multicolumn{3}{c}{ Rotation 1 } & \multicolumn{3}{c}{ Rotation 2 } \\
& $\ell$ & $b$ & $T_{\text {spot }}$ & $\ell$ & $b$ & $T_{\text {spot }}$ \\
\hline $\mathrm{A}$ & 20 & 35 & 4200 & 50 & 40 & 4050 \\
$\mathrm{~B}$ & 85 & 35 & 4250 & 110 & 35 & 4250 \\
$\mathrm{C}^{a}$ & 160 & $45-75$ & 3900 & 160 & $30-75$ & 3850 \\
$\mathrm{D}$ & 220 & 40 & 4050 & 230 & 35 & 4100 \\
$\mathrm{E}$ & 285 & 40 & 4250 & 280 & 35 & 4100 \\
$\mathrm{~F}$ & 340 & 30 & 4150 & 330 & 45 & 4100 \\
\hline
\end{tabular}

${ }^{a}$ This feature appears to be connected with the polar spot in both maps; its range in latitude is listed.

(i.e. mostly different $\log g$ and microturbulence) resulted in similar Doppler maps but with individual abundances different by up to \pm 0.2 dex.

\subsection{Doppler maps for two consecutive stellar rotations in 1996/97}

Figures 5a,b shows the average maps from three spectral regions and for two consecutive stellar rotations, respectively. One spectral region and two photometric bandpasses, $V$ and $I_{\mathrm{C}}$, are used simultaneously to produce a single map. Three such maps from Ca I 6439.08, Fe I 6430.84 and Fe I 6421.35 are then used to create an average map by averaging the temperature in each pixel. Each map is given equal weight because the overall $\chi^{2}$ achieved is similar. We caution though that the averaging may lead to an overemphasizing of the strongest lines because of their larger intrinsic line width and thus a more "smeared-out" temperature distribution relative to a weaker line. Therefore, we also present the individual maps in Figs. 6 and 7 for the two stellar rotations, respectively. All computations are performed on a DEC-Alpha $500 / 400$ workstation and require between 20 to $30 \mathrm{~min}$ of CPU time depending on the number of spectral blends, the number of input model atmospheres, and the number of photometric data points.

A visual comparison of the two consecutive maps in Figs. 5a and b reveals a comparable spot morphology as well as consistent absolute temperatures. A cool polar spot with an appendage centered around a longitude of $\ell \approx 160^{\circ}$ and a latitude of $\approx 60^{\circ}-70^{\circ}$ is seen in both maps (referred to as spot $\mathrm{C}$ in Table 3). Its lower end reaches almost down to the equator in Rotation 1 , but we believe that this is partly due to latitude smearing in the image reconstruction than a real single appendage and it is entirely possible that the feature consists of a high-latitude appendage and a low-latitude spot at the same longitude (the narrower Fe 6421 line shows actually two features well separated). The Fer 6421 map for the second rotation does not indicate the low-latitude spot anymore but instead show an enhanced polar appendage. One possible explanation could be that the lower spot had

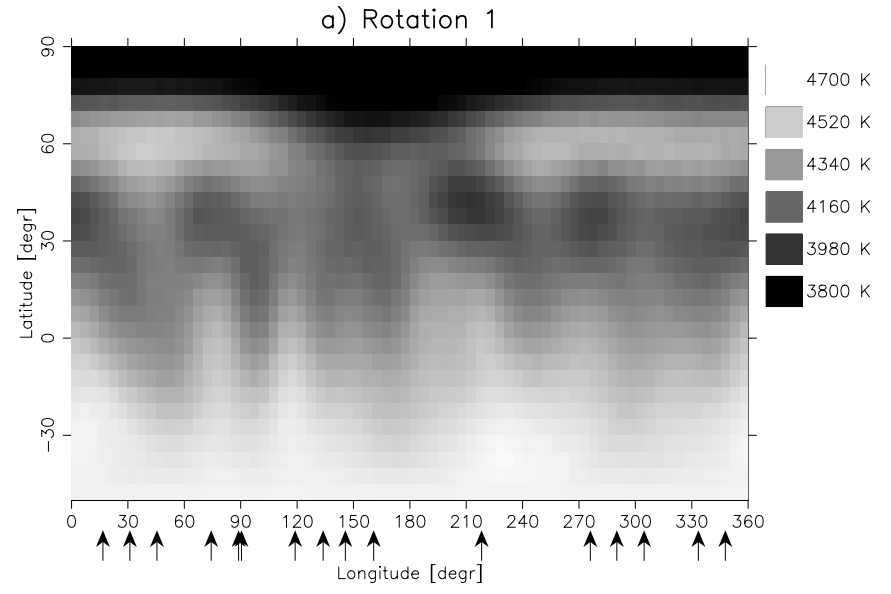

b) Rotation 2

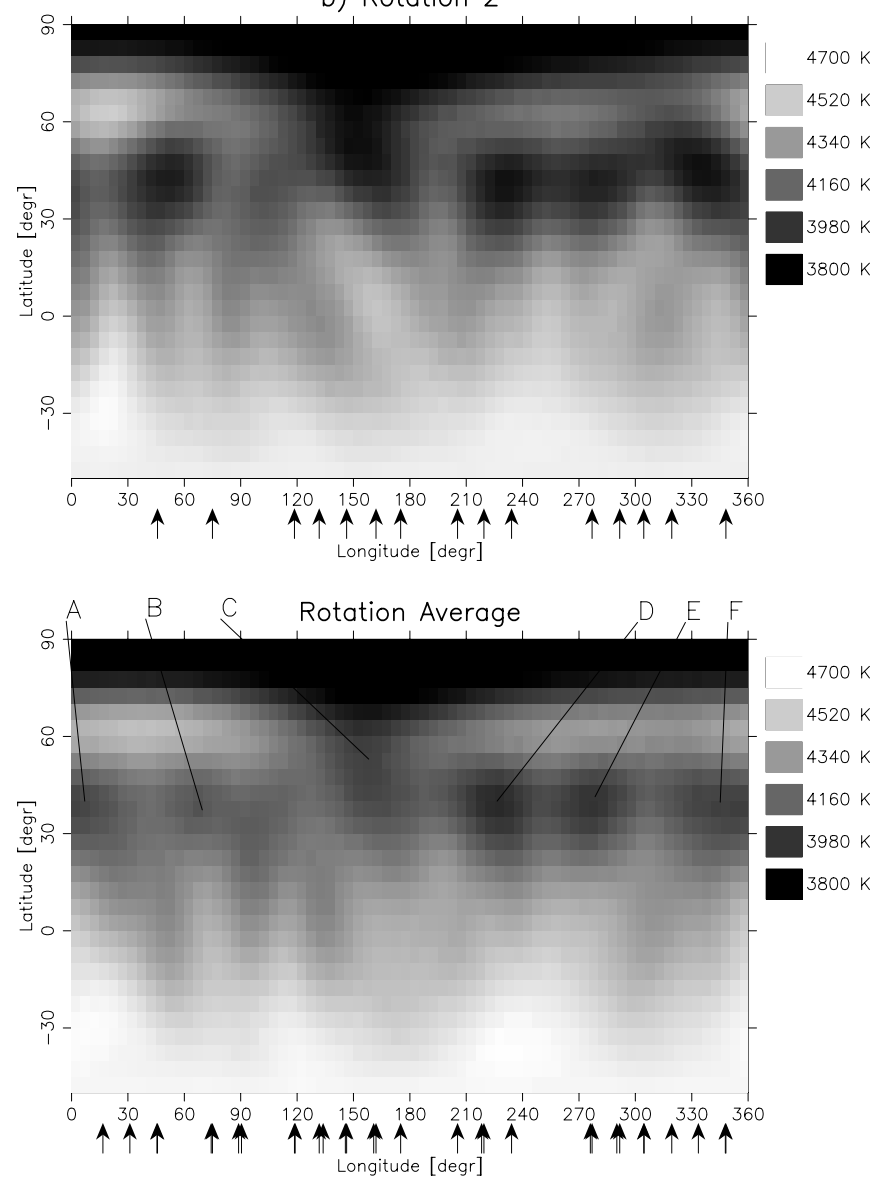

Fig. 5. Combined $\mathrm{Ca}$ and Fe maps for Rotation 1 a) and Rotation $2 \mathbf{b}$ ). The bottom map c) is computed from all spectral data combined and represents the time average for the two rotations. The rotation period is 25 days and the arrows below each map indicate the phases of the spectra.

merged with the polar appendage within a single stellar rotation.

Additionally to the polar spot there seem to be five spots at low-to-medium latitudes (named A, B, D, E, F from left to right in Fig. 5c). See Table 3 for a summary of the spot positions on both maps. Note that these numbers are measured off the maps by treating the Doppler 

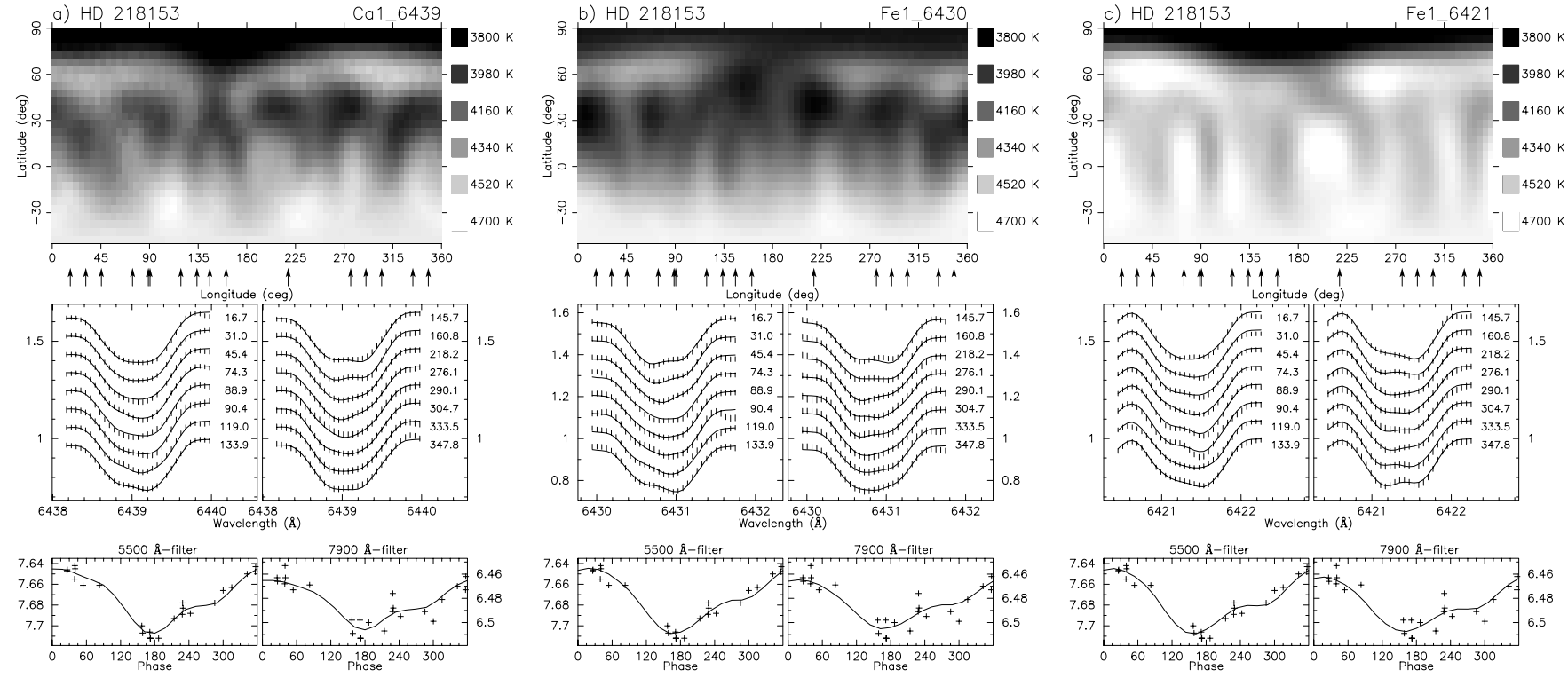

Fig. 6. Individual Doppler images for Rotation 1. a) Ca I $6439.08 \AA$ with an equivalent width of $272 \mathrm{~m} \AA$, b) Fe I $6430.84 \AA$ with $270 \mathrm{~m} \AA$, and c) Fe $6421.35 \AA$ with $221 \mathrm{~m} \AA$. Temperature maps from the individual lines are plotted in a pseudo-mercator (top row) projection where the arrows indicate the spectroscopic phase coverage. The observed and computed line profiles are shown in the middle row, and observed and computed $V$ and $I_{\mathrm{C}}$-lightcurves in the bottom row. The pluses and the bars are the observations and the full lines are the fits. The size of the bars correspond to a $\pm 1 \sigma$ error per data point.
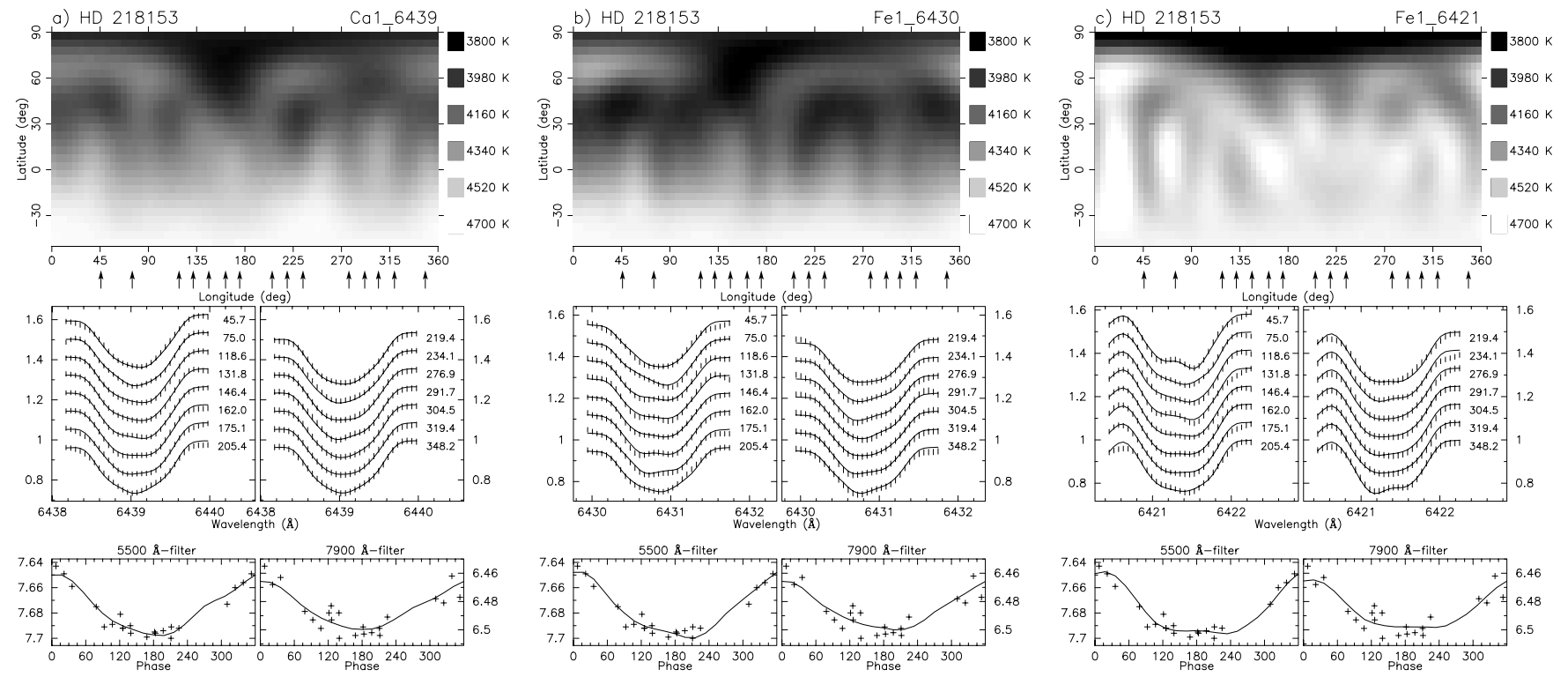

Fig. 7. Individual Doppler images for Rotation 2. Otherwise as in Fig. 6.

image as a CCD image in the CCD-photometry package digiphot in IRAF. We define a box around the approximate location of a feature and then use the IRAF routine phot to find a local (temperature) minimum within this box. The resulting coordinates have sub-pixel precision but a significantly worse accuracy that is determined by a large variety of external sources (see Rice \& Strassmeier 2000). We estimate an average of $\pm 5^{\circ}$ in latitude and somewhat less than that in longitude. The coolest of these spots has a temperature of about $800 \mathrm{~K}$ below the effective photospheric temperature of $4700 \mathrm{~K}$, and the warmest is approximately $450 \mathrm{~K}$ cooler. Typical errors for spot temperatures from TEMPMAP Doppler images are 70-100 K.

The equivalent widths of CaI $6439 \AA$ and Fe $6430 \AA$ are identical within the observational errors but the Fe I 6421 line is approximately $20 \%$ weaker. It is severely affected by the nearby Fe 6419 line and its large number of blends that affect the blue wing of the Fe 6421 line. Its fit to the observed light curves of the second rotation is also noticeably different to the fits from the other two lines. Despite this inconsistency, all spectral lines indicate a cool polar cap. The main difference in the maps is that 
the polar spot from the Fe 6421 line appears 100-150 K cooler than in the other two maps while the low-latitude spots appear warmer by that amount and, consequently, the polar spot's enhanced contrast seems eye catching. It is a known problem of line-profile inversions that a polar spots' size and contrast depends strongly on the fit to the line wings (see Unruh et al. 1996) and thus the contrast of the 6421-line is not a big surprise.

Nevertheless, to disentangle such inconsistencies, we compute a rotation-averaged map by combining all spectral data into one data set and invert it as usual. This map is shown in Fig. 5c; it is already the average from all three spectral regions. To quantify the differences between the fits of the individual rotations and the rotation-averaged fit, we used the average map to calculate synthetic line profiles in a forward solution and compare them with the inverse solutions from the two rotations. The difference of their $\chi^{2}$ is then an indirect measure for the reality of surface changes from one rotation to the next. Figure 8a summarizes these changes. Each individual point in this figure is the squared minimum of the residuals between a single observation and the rotation-averaged fit. We see, firstly, that the misfit is on average larger in the second rotation and, secondly, that there are two phases in both rotations with consistent $\Delta \chi^{2}$ peaks from all three spectral regions (at $\approx 100^{\circ}$ and $\approx 300^{\circ}$ ). The plots of the difference maps in Figs. $8 \mathrm{~b}$ and $\mathrm{c}$ finally show the changes on the stellar surface. We find that the asymmetry of the polar-spot appendage at $\approx 200^{\circ}$ had vanished in the second rotation and that two high-latitude regions appeared at $\approx 40^{\circ}$ and $\approx 300^{\circ}$. Two or maybe three of the lower-latitude spots seemed to have migrated. We interpret these differences to be due to real changes on the stellar surface and will investigate them further in the next chapter. For the FeI 6421 line profiles, the $\chi^{2}$ values in Fig. 8a are sometimes double than those for the two other lines. We thus consider the severely blended Fe I6421-line map more uncertain than the other two, albeit its overall $\chi^{2}$ is comparable and it recovers the features at approximately the same locations.

A further test of our Doppler images is to divide the spectra into "even" and "odd" data sets for each rotation, i.e. using first even-numbered spectra for an inverse solution and then the odd-numbered spectra, and then compare the resulting maps. The images from the rotationaveraged sets correlate very well, but the odd and even images for the individual rotations do not. The reason is that the phase coverage is just too sparse to derive results of the same quality as for the combined data set. Thus, the even-odd test is unfortunately not overly useful to verify the cross-correlation analysis applied in the next chapter.

\section{Differential surface rotation and meridional flows}

By cross correlating longitudinal strips at successive latitudes from the two maps in Figs. 5a, b, we can derive the differential surface rotation on KU Pegasi. Since the two maps are from consecutive rotations it is save to

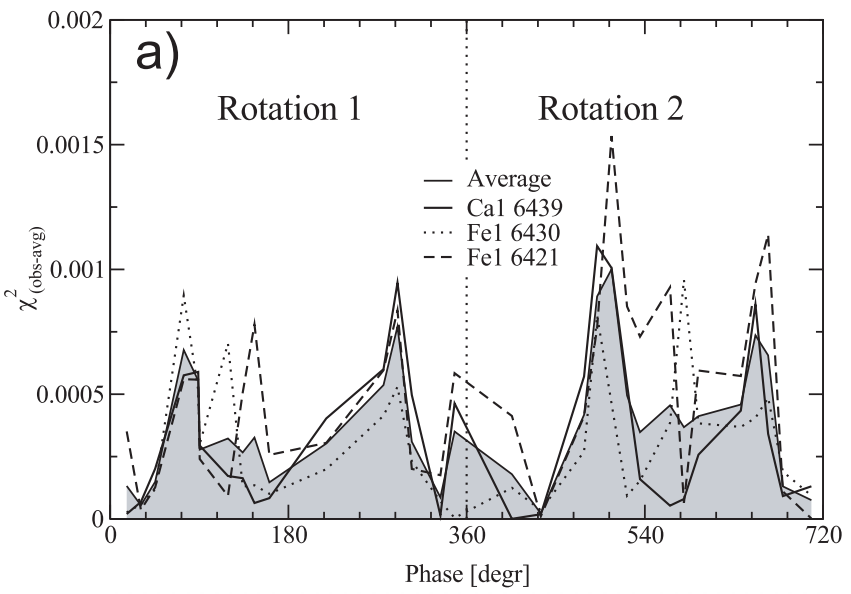

b) Rotation 1

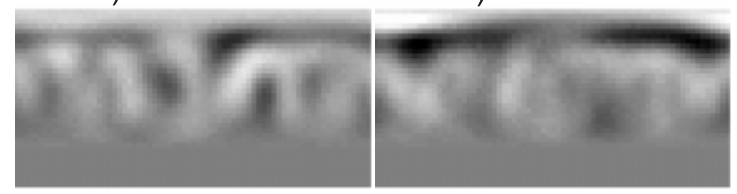

Fig. 8. a) The normalized goodness of fit for each observed line profile of the rotation-averaged Doppler-imaging solution. We use a $\chi^{2}$ statistic based on the three wavelength regions to obtain a single value for each spectral line profile. The photometry was not considered for this test. The grey area emphasizes the average from the three wavelength regions. A systematic deviation from zero is seen around two longitudes at $\approx 100^{\circ}$ and $\approx 300^{\circ}$, that suggests surface changes from one rotation to the next. b) and c) show the difference maps Rotation 1 minus rotation-averaged map and Rotation 2 minus rotationaveraged map, respectively (shown is just the average from all spectral lines).

assume that the surface features in the individual maps are still the same (but see previous section). In such a case the spots can be used as tracers for surface velocity fields, although the criterion $\tau_{\text {spot lifetime }}>\tau_{\text {observation }}$ is not necessarily a stringent requirement if there are many spots with the same general trend of migration. Of course, the interpretation is still hampered by the possibility of a coincidental spot alignment that mimics a latitude-dependent migration pattern. At this point, we simply caution the reader that our data are just a snapshot and will be masked by spot evolution. We applied the fxcor routine of the IRAF package (for details see the IRAF manual at iraf.noao.edu) to fit Gaussians to the peak of the cross-correlation functions for the Ca I 6439 image, the Fe I 6430 image, and the average of those two images. The result in Fig. 9 shows a complex surface differentialrotation function: the shifts within $\pm 25^{\circ}$ of the equator are tidily fitted with a solar-like differential-rotation law proportional to $\sin ^{2} b$, but between $+25^{\circ}$ and $+45^{\circ}$ (and possibly also between $-25^{\circ}$ and $-45^{\circ}$ ) the function changes its sign and thus these regions appear to accelerate again. Above $\approx+50^{\circ}$ the width of the cross-correlation function increases rapidly due to the decreased surface resolution and the longitudinal shifts cannot be measured very reliably there. Its error bars from the Gaussian fits 

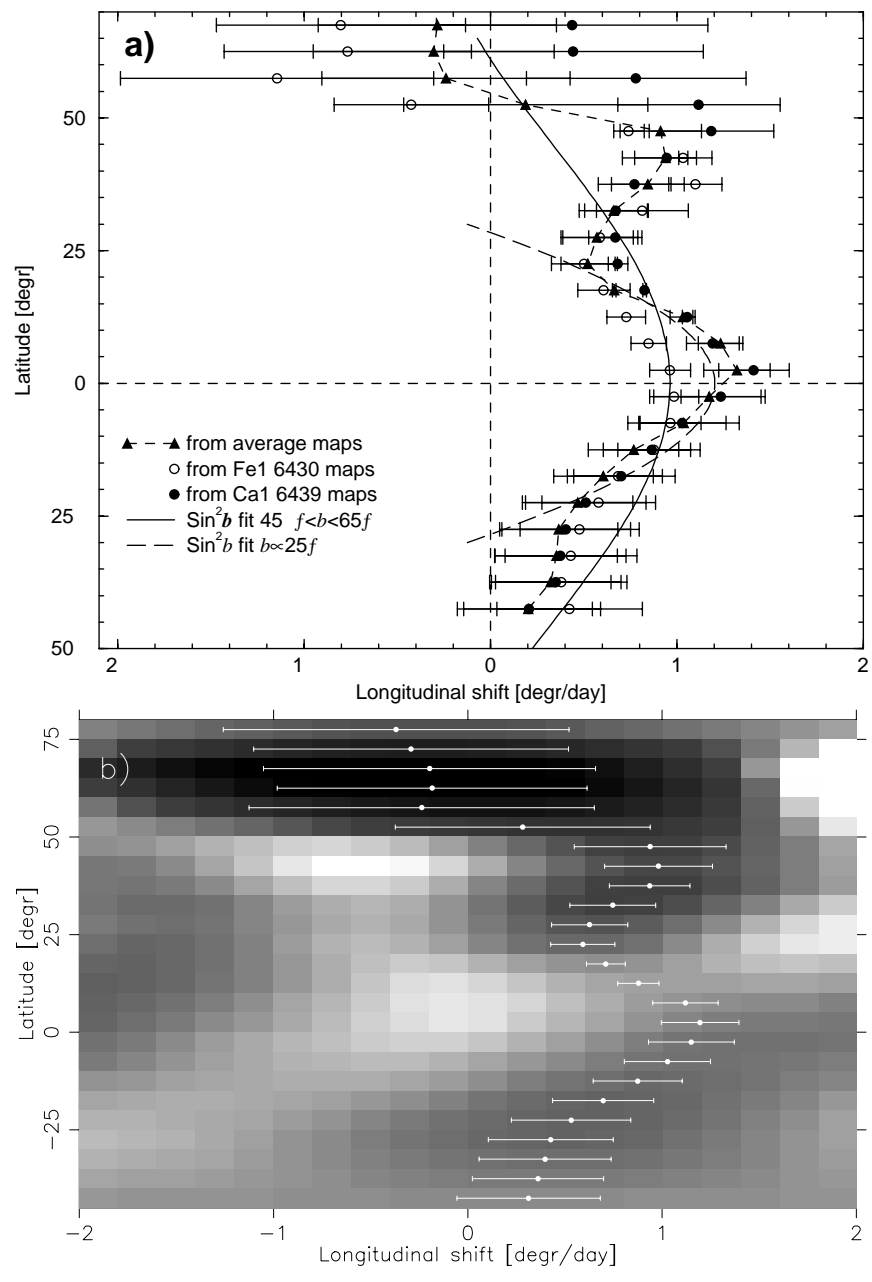

Fig. 9. Differential rotation on the surface of KU Peg. Plotted are the longitudinal shifts per latitude bin from a cross correlation of maps from Rotation 1 and 2. a) Symbols are the shifts measured and the lines are $\sin ^{2} b$-fits ( $b$ latitude) to the shifts. The full line is for the entire latitude range, the dashed line just for the equatorial $\pm 25^{\circ}$ range. b) The average of the shifts derived from individual lines on top of a greyscale image of the cross correlation of the two average maps. The phase lag of the higher latitude regions possibly indicates that higher-latitude regions rotate slower than the equator.

are $\approx 3$ times larger than near the equator. Despite this limitation there is some evidence though that the rotation decelerates again above $\approx+50^{\circ}$. This is certainly inconclusive from our two stellar rotations but should not go unnoticed.

We tried to fit the cross correlations with a simple $\sin ^{2} b$ law for the entire latitude range (full line in Fig. 9). Such a fit is obviously not a good representation of the data (rms of 0.29) but is intended to obtain a save lower limit for the magnitude of the differential rotation. We also did a $\sin ^{2} b$ fit restricted to shifts within $\pm 25^{\circ}$ latitude (dashed line in Fig. 9) and thereby also obtain an estimate of the external error per latitude range. Its rms is accordingly better, 0.11 . The first fit leads to the following
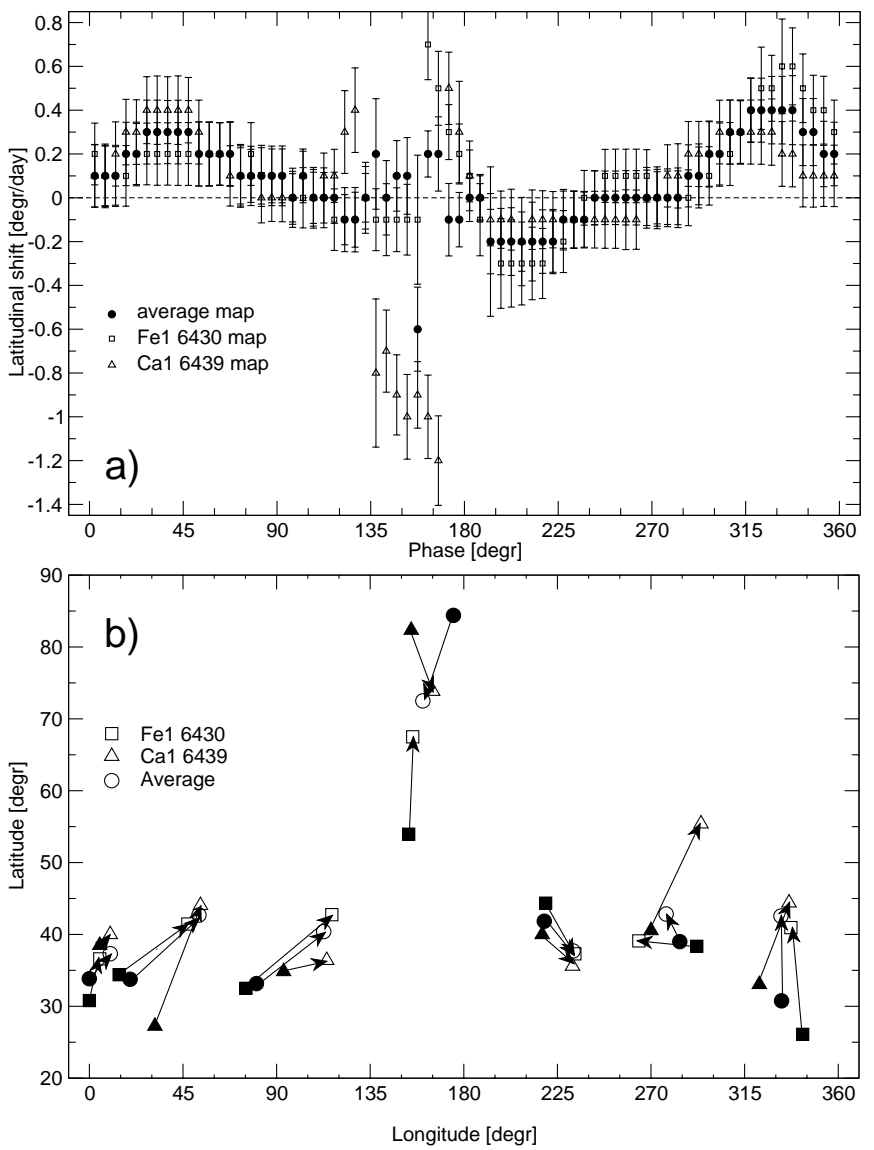

Fig. 10. a) Meridional changes on the surface of KU Peg. Plotted are the latitudinal shifts per longitudinal bin from a cross correlation of the hemisphere above the equator from Rotation 1 and 2 . The two bumps at $40^{\circ}$ and $330^{\circ}$ possibly indicate a poleward flow of the magnitude of $\approx 0.4^{\circ}$ days $^{-1}$. b) The redistribution of spots from Rotation 1 (filled symbols) to Rotation 2 (open symbols). The individual spots are listed in Table 3.

differential rotation law for $-40^{\circ}$ to $65^{\circ}$

$$
\begin{aligned}
\Omega(b) & =\Omega_{0}-\Omega_{1} \sin ^{2} b \\
& =15.41-1.38 \sin ^{2} b \quad\left[{ }^{\circ} \mathrm{day}^{-1}\right] .
\end{aligned}
$$

The second fit for $\pm 25^{\circ}$ gives

$$
\begin{aligned}
\Omega(b) & =\Omega_{0}-\Omega_{1} \sin ^{2} b \\
& =15.63-5.32 \sin ^{2} b \quad\left[{ }^{\circ} \mathrm{day}^{-1}\right] .
\end{aligned}
$$

The differential rotation coefficient $\alpha=\left(\Omega_{0}-\Omega_{\text {pole }}\right) / \Omega_{0}=$ $\Omega_{1} / \Omega_{0}$ is from Eq. $(2) \approx 0.09$ and $1 / \Delta \Omega$, i.e. the time the equatorial region laps the pole, $\approx 260$ days. This is comparable to the solar value of 120 days. If we only take the latitudes below $\pm 25^{\circ}$ into account (Eq. (3)), we get values of $\alpha=0.34$ and $1 / \Delta \Omega \approx 70$ days.

To quantify latitudinal changes on the stellar surface, we now cross correlate the maps along meridional circles. We just adopt the "northern" hemisphere, i.e. the hemisphere that is fully in view (all pixels with positive latitude), and cross correlate its longitudinal strips of the CaI 6439 images, the Fe I 6430 images, and the 

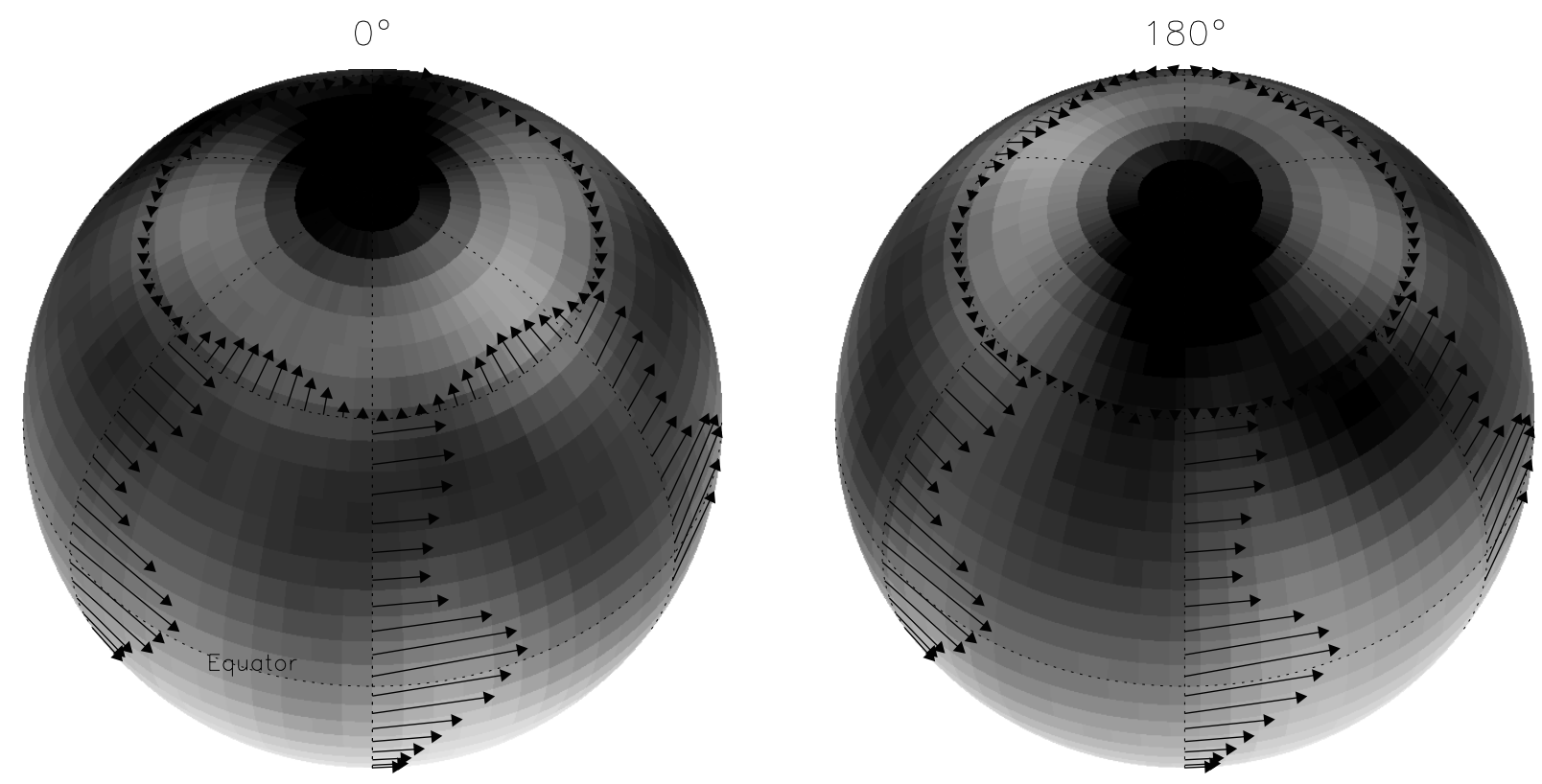

Fig. 11. The average Doppler image from the first rotation in orthographic projection at phase zero (left) and phase $180^{\circ}$ (right). The size and the direction of the arrows indicate the surface flows resulting from our cross correlations. The longitudes and latitudes at which the arrows are drawn are arbitrary because the net shifts were computed from all longitudes and latitudes $\left(\ell=0^{\circ}\right.$ and $\pm 60^{\circ}$, and $b=50^{\circ}$ are shown here). Note that the poleward flow is strongest on the opposite side of the polar-spot appendage.

average of those two images, respectively. The result in Fig. 10a clearly shows that there were two latitudinal shifts on the surface of KU Peg within one stellar rotation that consistently appeared in both spectral lines. One event at phase $\approx 0.1\left(40^{\circ}\right)$ and another at $\approx 0.9\left(330^{\circ}\right)$, with an average magnitude of $+0.3 \pm 0.1(\mathrm{rms})^{\circ}$ day $^{-1}$ and $+0.4 \pm 0.1(\mathrm{rms})^{\circ}$ day $^{-1}$, respectively. The intermittent longitudes on the opposite side of the visible pole may show a reversed shift of magnitude $-0.2^{\circ}$ day $^{-1}$, but this may be overinterpretation given the large error bars and the inconsistency at phases from approximately $135^{\circ}$ to $180^{\circ}$. The latter is most likely caused by the wellknown "north-south" mirroring of the polar appendage at $\ell \approx 160^{\circ}$.

As for the previous cross correlations the error bars per bin are estimated from a Gaussian fit of the FWHM of the cross correlation function. Both shifts have a positive sign and thus indicate a polar-directed change. We interpret these shifts as a meridional change of magnetic flux and, since it seems to be a local event on the stellar surface, tentatively suggest that magnetic reconnection and its associated plasma motions may be the underlying cause rather than a global meridional flow.

\section{Discussion and summary}

We detected a complex surface differential-rotation law for KU Peg with acceleration along the equator and possibly also near a latitude of $\approx 50^{\circ}$, and deceleration in between and above. KU Peg's lap time $1 / \Delta \Omega$ is $\approx 70$ days for the $\pm 25^{\circ}$ range around the equator, but 260 days for the entire equator-to-pole range. This differs from the solar case and from the findings of Collier Cameron et al. (2001) for the three ultra-fast rotators RX J1508 - 4423 (G2), AB Dor $(\mathrm{K} 0 / 2 \mathrm{~V})$, and PZ Tel $(\mathrm{K} 0 \mathrm{IV} / \mathrm{V})$ with rotation periods of $0.31,0.515$, and 0.94 days, respectively, in that these stars show a uniform differential rotation that follows a simple solar-like $\sin ^{2} b$ law. Their lap times $1 / \Delta \Omega$ are 40,110 , and 80 days, respectively, which are all shorter than the solar value of 120 days. LO Peg (K5-7V, $P_{\text {rot }}=0.424$ days $)$ on the other hand, does show signs for the equator lagging behind (Lister et al. 1999). These authors argue though that the absence of mid-latitude features together with the short time between the two images (one day) possibly prevents the detection of significant differential rotation. Rice \& Strassmeier (1996) detected differential rotation on the weak-lined T Tauri star V410 Tau $\left(P_{\text {rot }}=1.87\right.$ days $)$ in the same sense as on the Sun but with a laptime of 1800 days, a factor of 15 weaker, which is significantly different from the stars above. Either each star with a given mass, rotation period, and evolutionary status has its own distinct differential surface rotation, as e.g. indicated by the theoretical models from Kitchatinov \& Rüdiger (1999), or the observations are masked by local and rapid magnetic-field reconfigurations that just mimic a differential rotation law.

Differential rotation was reported for several other evolved stars: Weber \& Strassmeier (1998) found equatorial acceleration on the RS CVn binary IL Hya for latitudes below $45^{\circ}$ and about a factor of 30 smaller than on the Sun, as measured from two images taken $\approx 28$ stellar rotations apart. Strassmeier (1994) and Hatzes (1998) derived a differential-rotation law for HU Vir (also a RS CVn star) from the comparison of two temperature maps taken 
2 stellar rotations and 4 years apart, respectively, and combined with contemporaneous photometric modeling included in Strassmeier (1994). Both authors found differential rotation of inverse behavior than on the Sun polar regions rotate faster than low-latitude regions - and a factor of 10 slower. However, the spot features used in both studies were all at $b \geq 30^{\circ}$ and thus no reliable information for the equatorial region was available. Hatzes \& Vogt (1992) found solar-like differential rotation on the short-period (1.95 days) RS CVn binary EI Eri, i.e. equatorial acceleration and similarity in strength, but an inverse behavior, and about a factor of 10 smaller than on the Sun, on the 6.4-days RS CVn binary UX Ari (Vogt \& Hatzes 1991).

So far, there is cumulative evidence that differential rotation profiles on evolved stars (and possibly also on pre-main-sequence stars) appear to be more complicated than on solar-type main-sequence or ZAMS stars. A recent study of the RS CVn binary HR 1099 by Strassmeier \& Bartus (2000) reveals a general poleward spot migration of the order of $0.4^{\circ}$ day $^{-1}$ simultaneously to longitudinal spot migrations with both signs at the same time, i.e. spots migrating faster and slower than the orbital period but are located at approximately the same latitude. This is in agreement with an earlier claim by Vogt et al. (1999) based on 23 Doppler images taken throughout 11 years. KU Peg also shows evidence, like HR 1099, for poleward spot migration and even of the same amount. There is also some similarity of our KU Peg result to the recently obtained rotation profile for the rapidly-rotating long-period K0III binary $\sigma$ Gem $\left(P_{\text {rot }} \approx 20\right.$ days, Kövári et al. 2001). For $\sigma$ Gem, they found a differential rotation law in quadratic form with acceleration in two latitudinal bands centered at approximately $\pm 40^{\circ}$ around the equator, but deceleration along the equator and near the one visible pole. We believe that all of these observations hint toward a general dependence of differential rotation upon rotational period. Giants seem to show a mixture of solar-like and anti-solar profiles of various strengths, which seems to be partially in conflict with the recent differential-rotation models of Kitchatinov \& Rüdiger (1999) who predict larger differential rotation in giants than in dwarfs. It is also indicative that differential rotation is not the only way to explain spot migrations and that the associated meridional flow may play a stronger role on giants than, e.g., for the Sun.

The Sun, for comparison, has a very weak latitudinal flow pattern of $\pm 0.03^{\circ}$ day $^{-1}$ (Howard \& Gilman 1986). This flow transports magnetic flux from mid-latitude spots up to the rotation poles where its opposite polarity causes the polarity reversal and the end of an old magnetic cycle and the start of a new one. So far, stellar observations of a poleward flow exist only for stars with high-latitude active regions but are in agreement with the picture first presented by Schüssler \& Solanki (1992). In that picture, the flux tubes can arise at latitudes up to $\approx 60^{\circ}$ if the star rotates rapidly enough. However, an additional transportation mechanism is necessary to move the spot towards the pole once it has emerged. This is different from the very young stars, where a truly polar spot can emerge without the additional need of a meridional flow (see Granzer et al. 2000 for a recent discussion).

For some of the previously discussed stars, the time between the individual maps was usually many rotations, and thus spot changes with timescales less than a few rotations could not be determined. Only spots close to the poles seem to be persistent enough to be seen throughout many rotations and this may bias our meridional-flow detections. However, if some mechanism does transport active regions towards the pole, where they make up for a large torodial field that, in return, inhibits differential rotation, then the difficulty of detecting differential rotation on such stars is not a surprise. One such star is the 16-day G8II-III giant CM Cam (Strassmeier et al. 1998), where cross correlations of Doppler images from four observing seasons with one year in between did not reveal a clear differential-rotation signal, despite that there is evidence for phase shifts on its surface. A very similar case is the single G5 giant HD 199178 (Strassmeier et al. 1999b), where images taken one month and images taken one year apart were cross-correlated but no systematic migration pattern was found. Whether the time the magnetic field needs to reconfigure on these stars is too short to be detected or, whether an existing differential rotation pattern is simply masked by short-term field configuration changes, could not be answered in those two cases.

Acknowledgements. Thanks to Trudy Tilleman for operating the McMath telescope half of the time and for providing gourmet coffee all of the time, to the Austrian Fond zur Förderung der wissenschaftlichen Forschung (FWF) for support under grants S7301-AST and S7302-AST, and to the German Forschungsgemeinschaft (DFG) for grant HU 532/8. We thank the referee, Dr. J. R. De Medeiros, for his constructive criticism.

\section{References}

Barden, S. C. 1985, ApJ, 295, 162

Barrado y Navascues, D., De Castro, E., Fernandez-Figueroa, M. J., Cornide, M., \& Garcia Lopez, R. J. 1998, A\&A, 337, 739

Bell, R. A., \& Gustafsson, B. 1989, MNRAS, 236, 653

Bidelman, W. P. 1983, AJ, 88, 1182

Bopp, B. W., Dempsey, R. C., \& Maniak, S. 1988, ApJS, 68, 803

Collier Cameron, A., Barnes, J. R., Kitchatinov, L., \& Donati, J.-F. 2001, in Eleventh Cambridge Workshop on Cool Stars, Stellar Systems and the Sun, ed. R. G. Lopez, R. Rebolo, \& M. R. Zapatero Osorio, ASP Conf. Ser., 223, 251

De Medeiros, J. R., Mayor, M., \& Simon, T. 1992, A\&A, 254, L36

Dyck, H. M., van Belle, G. T., \& Thompson, R. R. 1998, AJ, 116,981

ESA 1997, The Hipparcos Catalogue, ESA SP-1200

Fekel, F. C. 1997, PASP, 109, 514

Fekel, F. C., \& Balachandran, S. 1993, ApJ, 403, 708

Fernie, J. D. 1983, ApJS, 52, 7

Flower, P. J. 1996, ApJ, 469, 355 
Granzer, T., Schüssler, M., Caligari, P., \& Strassmeier, K. G. 2000, A\&A, 355, 1087

Guarinos, J. 1992, in Astronomy from large databases II, ed. A. Heck, \& F. Murtagh, ESO Conf. Workshop Proc., 43, 301

Guetter, H. H. 1980, PASP, 92, 215

Hatzes, A. P. 1998, A\&A, 330, 541

Hatzes, A. P., \& Vogt, S. S. 1992, MNRAS, 258, 387

Howard, R., \& Gilman, P. A. 1986, ApJ, 307, 389

Kitchatinov, L. L., \& Rüdiger, G. 1999, A\&A, 344, 911

Kővári, Zs., Strassmeier, K. G., Bartus, J., et al. 2001, A\&A, submitted (Paper XV)

Kupka, F., Piskunov, N., Ryabchikova, T. A., Stempels, H. C., \& Weiss, W. W. 1999, A\&AS, 138, 119

Kurucz, R. L. 1993, ATLAS-9 CD ROM, No. 13

Lister, T. A., Collier Cameron, A., \& Bartus, J. 1999, MNRAS, 307,685

Piskunov, N. E., \& Rice, J. B. 1993, PASP, 105, 1415

Rice, J. B. 1996, in Stellar Surface Structure, ed. K. G. Strassmeier, \& J. L. Linsky, IAU Symp. 176 (Kluwer Academic Publishers Dordrecht), 19

Rice, J. B., \& Strassmeier, K. G. 1996, A\&A, 316, 164 (Paper II)

Rice, J. B., \& Strassmeier, K. G. 2000, A\&AS, 147, 151

Rice, J. B., Wehlau, W. H., \& Khokhlova, V. L. 1989, A\&A, 208, 179

Schaller, G., Schaerer, D., Meynet, G., \& Maeder, A. 1992, A\&AS, 96, 269

Schmidt-Kaler, T. 1982, in Landolt-Börnstein, vol. 2b, ed. K. Schaifers, \& H. H. Voigt (Springer Verlag Berlin Heidelberg New York)

Schüssler, M., \& Solanki, S. K. 1992, A\&A, 264, L13

Sperl, M. 1998, Comm. Astroseismol., 111
Strassmeier, K. G. 1994, A\&A, 281, 395

Strassmeier, K. G., \& Bartus, J. 2000, A\&A, 354, 537 (Paper XII)

Strassmeier, K. G., Bartus, J., Kővári, Zs., Weber, M., \& Washuettl, A. 1998, A\&A, 336, 587 (Paper VIII)

Strassmeier, K. G., Boyd, L. J., Epand, D. H., \& Granzer, T. 1997, PASP, 109, 697

Strassmeier, K. G., Fekel, F. C., Bopp, B. W., Dempsey, R. C., \& Henry, G. W. 1990, ApJS, 72, 191

Strassmeier, K. G., \& Hall, D. S. 1988, ApJS, 67, 453

Strassmeier, K. G., Lupinek, S., Dempsey, R. C., \& Rice, J. B. 1999b, A\&A, 347, 212 (Paper X)

Strassmeier, K. G., \& Rice, J. B. 1998, A\&A, 330, 685 (Paper VI)

Strassmeier, K. G., \& Schordan, P. 2000, Astr. Nachr., 321, 277

Strassmeier, K. G., Serkowitsch, E., \& Granzer, T. 1999a, A\&AS, 140, 29

Strassmeier, K. G., Washuettl, A., Granzer, T., Scheck, M., \& Weber, M. 2000, A\&AS, 142, 275

Unruh, Y. C. 1996, in Stellar Surface Structure, IAU Symp. 176, ed. K. G. Strassmeier, \& J. L. Linsky (Kluwer Academic Publishers Dordrecht), 35

van Belle, G. T., Lane, B. F., Thompson, R. R., et al. 1999, AJ, 117, 521

Vogt, S. S., \& Hatzes, A. P. 1991, in IAU Colloq. 130: The Sun and Cool Stars. Activity, Magnetism, Dynamos, ed. I. Tuominen, D. Moss, \& G. Rüdiger (Springer Verlag Berlin), 297

Vogt, S. S., Hatzes, A. P., Misch, A. A., \& Kürster, M. 1999, ApJS, 121, 547

Weber, M., \& Strassmeier, K. G. 1998, A\&A, 330, 1029 (Paper V) 